\title{
Design and Pharmaceutical Evaluation of a Nano-Enabled Crosslinked Multipolymeric Scaffold for Prolonged Intracranial Release of Zidovudine
}

Sheri-lee Harilall ${ }^{1}$, Yahya E. Choonara ${ }^{1}$, Girish Modi ${ }^{2}$, Lomas K. Tomar ${ }^{1}$, Charu Tyagi ${ }^{1}$, Pradeep Kumar ${ }^{1}$, Lisa C. du Toit $^{1}$, Sunny E. Iyuke ${ }^{3}$, Michael P. Danckwerts ${ }^{1}$ and Viness Pillay ${ }^{1}$

${ }^{1}$ University of the Witwatersrand, Faculty of Health Sciences, Department of Pharmacy and Pharmacology, 7 York Road, Parktown, Johannesburg, South Africa. ${ }^{2}$ University of the Witwatersrand, Department of Neurology, Division of Neurosciences, Johannesburg, South Africa. ${ }^{3}$ University of the Witwatersrand, School of Chemical and Metallurgical Engineering, Johannesburg, South Africa.

Received, May 9, 2013; Revised, June 18, 2013; Accepted, July 9, 2013; Published, July 11, 2013.

\begin{abstract}
Purpose. Nanomedicine explores and allows for the development of drug delivery devices with superior drug uptake, controlled release and fewer drug side-effects. This study explored the use of nanosystems to formulate an implantable drug delivery device capable of sustained zidovudine release over a prolonged period. Methods. Pectin and alginate nanoparticles were prepared by applying a salting out and controlled gelification approach, respectively. The nanoparticles were characterized by attenuated total reflectance-fourier transform infrared spectroscopy (ATR-FTIR), scanning electron microscopy (SEM), transmission electron microscopy (TEM) and dynamic light scattering (DLS) and were further evaluated for zidovudine (AZT) entrapment efficiency. Multipolymeric scaffolds were prepared by crosslinking carboxymethyl cellulose, polyethylene oxide and epsilon caprolactone for entrapment of zidovudine-loaded alginate nanoparticles to impart enhanced controlled release of zidovudine over the time period. Swelling and textural analysis were conducted on the scaffolds. Prepared scaffolds were treated with hydrochloric acid $(\mathrm{HCl})$ to reduce the swelling of matrix in the hydrated environment thereby further controlling the drug release. Drug release studies in phosphate buffered saline $\left(\mathrm{pH} 7.4,37^{\circ} \mathrm{C}\right)$ were undertaken on both zidovudine-loaded nanoparticles and native scaffolds containing alginate nanoparticles. Results. A higher AZT entrapment efficiency was observed in alginate nanoparticles. Biphasic release was observed with both nanoparticle formulations, exhibiting an initial burst release of drug within hours of exposure to PBS, followed by a constant release rate of AZT over the remaining 30 days of nanoparticle analysis. Exposure of the scaffolds to $\mathrm{HCl}$ served to reduce the drug release rate from the entrapped alginate nanoparticles and extended the AZT release up to 30 days. Conclusions. The crosslinked multipolymeric scaffold loaded with alginate nanoparticles and treated with $1 \% \mathrm{HCl}$ showed the potential for prolonged delivery of zidovudine over a period of 30 days and therefore may be a potential candidate for use as an implantable device in treating Aids Dementia Complex.
\end{abstract}

This article is open to POST-PUBLICATION REVIEW. Registered readers (see "For Readers") may comment by clicking on ABSTRACT on the issue's contents page.

\section{INTRODUCTION}

The National Science Foundation in the United States of America (USA) estimates that by 2015, the annual global market for nanotechnologicalrelated goods and services will reach $\$ 1$ trillion. It has also been estimated that by $2014,16 \%$ of healthcare and life sciences related commodities will incorporate nanotechnology, making it one of the fastest growing industries in history, and an exceptionally large economic force (1). Nanotechnology offers much promise for the enhancement of disease diagnosis, management and prevention (2,3). Drug incorporation into nanosystems is used to achieve site-specific drug delivery, providing improved control of drug release which augments the bioavailability, efficacy, pharmacokinetics and pharmacodynamics of the drug. Nanostructures offer drug release for desired time periods, thereby improving therapeutic efficacy and reducing drug toxicity $(2,4,5)$. Nanoparticles can even be used as carriers for poorly soluble drugs, acting to augment bioavailability of such drugs $(6,7,8)$.

Corresponding Author: Professor Viness Pillay; University of the Witwatersrand, Faculty of Health Sciences, Department of Pharmacy and Pharmacology, 7 York Road, Parktown, Johannesburg, South Africa; Email: viness.pillay@wits.ac.za 
Nanocarriers are made from an assortment of organic and inorganic materials including nondegradable and biodegradable polymers, lipids, self-assembling amphiphilic molecules, dendrimers, metal, and inorganic semiconductor nanocrystals (2). Biocompatible and biodegradable polymers with desirable physicochemical and physicomechanical properties have been researched extensively for the formulation of novel drug delivery systems. Alginate is one such hydrophillic natural polysaccharide of algal origin, which is biocompatible, biodegradable and non-toxic in nature. It comprises of linear chains of $\alpha-1$ glucuronic acid (G) and (1-4)-linked $\beta$-Dmannuronic acid $(\mathrm{M})$ residues, which vary in composition and sequence $(9,10,11)$. Alginate forms insoluble gels in the presence of bivalent calcium $\left(\mathrm{Ca}^{2+}\right)$ ions and other multivalent metals by binding to the carboxylic groups of adjacent $\mathrm{G}$ units in the polymer. This $\mathrm{Ca}^{2+}$-alginate gel is frequently used as drug delivery vehicle $(10,11)$. Rajaonarivony et al., in 1993 developed a method for the preparation of alginate nanoparticles using a novel cation induced pre-gelation of alginate approach (12). This method has since been adapted by many scientists for the preparation of alginate nanoparticles, with chitosan being used instead of poly-L-lysine which is a cationic natural polymer but toxic and immunogenic if injected. These nanoparticles have been used as carrier for a variety of drugs, including insulin, chemotherapeutics, as well as the hydrophobic antihypertensive agent, Nifedipine $(9,10,13-17)$. Pectin is another linear polysaccharide of plant origin, derived by aqeuos extraction of edible plants, mainly apples and citrus fruits $(10,18,19)$. It is a biocompatible, biodegradable, stable, nontoxic and hydrophillic natural polymer mainly consisting of $\alpha$-( $1 \rightarrow 4)$-D-galacturonic acid residues arranged in a linear fashion $(10,18-20)$. Like alginate pectin forms gel in the presence of $\mathrm{Ca}^{2+}$ ions, sugars and acids and is therefore utilised in the preparartion of various food and pharmaceutical products. Sodium carboxymethyl cellulose $(\mathrm{NaCMC})$ is a carboxymethyl ether of cellulose, a naturally occurring plant polysaccharides present in the fibrous tissue of all plants. The polymer exhibits high water solubility attributed to the introduction of sodium carboxymethyl groups into the cellulose molecule, by etherification of the hydroxyl groups of glucopyranose unit $(18,21-24)$. NaCMC is often utilised pharmaceutically as a viscosity enhancer in oral, parental and topical formulations $(18,23)$.
Epsilon caprolactone (ECL) is synthetic, semi-crystalline aliphatic polyester, whose properties can be altered by catalysed ringopening copolymerisation. ECL has been employed for the preparation of many sustained release drug delivery formulations due to its slow rate of degradation, as well as superior drug permeability (25-28). Polyethylene oxide (PEO) is a semi-crystalline water soluble polymer. The hydrophilic hydroxyl $(\mathrm{OH})$ groups present in the molecule, allows for bioadhesivity and mucoadhesivity of the polymer. PEO is frequently used for oral drug delivery due to its ability to swell and form a gel layer upon exposure to water, thereby controlling drug release as a result of retarded erosion of the formulation $(29,30)$.

The novelty of this study involves the use of the polymers described above to prepare nanoparticles and a crosslinked multipolymeric scaffold for prolonged drug delivery of the model drug zidovudine (AZT) as a biodegradable, biocompatible and non-cytogenic device for the potential intracranial treatment of Aids Dementia Complex (ADC). The by-products of these polymers are non-toxic and readily excreted from the body (7,27,31). They possess desirable mechanical properties and superior drug permeability and are therefore safe for use in the human body. $(7,27,28,31,32)$. Nanoparticles were prepared using two methods, the salting-out approach and the controlled gelification of alginate approach. Suitable nanoparticles were then dispersed within a multipolymeric scaffold, prepared by means of polymer crosslinking to obtain a drug delivery device capable of sustained drug release over a period of at least 30 days.

\section{METHODS}

\section{Materials}

Alginate, pectin, polyethylene oxide (PEO), polyvinyl alcohol (PVA), sodium carboxymethyl cellulose (NaCMC) and epsilon caprolactone (ECL) were purchased from Sigma Aldrich (St Louis, MO, USA). These polymers were utilized to prepare nanoparticles and the polymer scaffold. Calcium chloride $\left(\mathrm{CaCl}_{2}\right)$, zinc sulphate solution $\left(\mathrm{ZnSO}_{4}\right)$, aluminium chloride $\left(\mathrm{AlCl}_{3}\right)$ and sodium thiosulphate $\left(\mathrm{Na}_{2} \mathrm{~S}_{2} \mathrm{O}_{3}\right)$ were also purchased from Sigma Aldrich (St Louis, MO, USA) and were used as the crosslinking agents for the synthesis of the nanoparticles and the multipolymeric scaffold. Zidovudine (AZT) used as the model drug and was purchased from Evershine Ind., (Naejar Malad, Mumbai, India). All buffers were prepared using Milli-Q water. 
Preparation of the polymeric nanoparticles Pectin nanoparticles prepared by the saltingout approach

Pectin nanoparticles were prepared by employing a salting-out approach whereby a polymeric dispersion was formed by agitating $0.8 \% \mathrm{w} / \mathrm{v}$ pectin $(50 \mathrm{~mL})$ with a $24 \% \mathrm{w} / \mathrm{v} \mathrm{ZnSO}_{4}$ solution containing $0.6 \mathrm{~g}$ PVA $(75 \mathrm{~mL})$ that was used as the salting-out reagent. The rapid addition of deionized water to the polymeric dispersion induced the formation of nanoparticles which were subsequently lyophilized for 24 hours. In situ drug-loading of AZT was accomplished by dissolving the drug in the pectin solution prior to the addition of the crosslinking solution.

\section{Alginate nanoparticles prepared by controlled gelification}

Nanoparticles were also prepared by ionotropic gelification of anionic sodium alginate $(0.6 \% \mathrm{w} / \mathrm{v})$ $(200 \mathrm{~mL})$ with cationic $\mathrm{CaCl}_{2}(10 \mathrm{~mL})$ after which a $0.5 \% \mathrm{w} / \mathrm{v}$ pectin solution $(40 \mathrm{~mL})$ was added to the $\mathrm{Ca}^{2+}$ alginate gel to form a stable nanoparticle colloidal system. The crosslinked solution was then rapidly stirred for 1 hour and thereafter centrifuged (Optima ${ }^{\circledR}$ LE-80K, Beckman, USA) at $20,000 \mathrm{rpm}$ for 15 minutes. The supernatant was then removed and lyophilized for 24 hours at 25mtorrs (Virtis, Gardiner, NY, USA) to obtain a free flowing powder after having refrigerated the sample at $-70^{\circ} \mathrm{C}$. AZT-loaded nanoparticles were prepared similarly with $0.3 \mathrm{~g}$ of AZT dissolved in the alginate solution prior to $\mathrm{CaCl}_{2}$ addition to enable encapsulation of the drug within a polymeric core.

\section{Preparation of the crosslinked multipolymeric scaffold}

Polymer solutions of $\mathrm{NaCMC}(3 \% \mathrm{w} / \mathrm{v}, 100 \mathrm{~mL})$, PEO $(1 \% \mathrm{w} / \mathrm{v}, 100 \mathrm{~mL})$ and ECL $(5 \% \mathrm{w} / \mathrm{v}, 100 \mathrm{~mL})$ were blended until a homogenous mixture was obtained followed by the dispersion of the AZTloaded alginate nanoparticles $(7.265 \mathrm{~g})$ into the multipolymeric solution. The polymeric mixture, in $5 \mathrm{~mL}$ increments, was then placed into Teflon moulds (measuring $15 \mathrm{~mm}$ in diameter and $20 \mathrm{~mm}$ in height) lubricated with liquid paraffin, containing $2 \mathrm{~mL}$ of a $10 \% \mathrm{w} / \mathrm{v} \mathrm{AlCl}_{3}, \mathrm{CaCl}_{2}$ and $\mathrm{Na}_{2} \mathrm{~S}_{2} \mathrm{O}_{3}$ crosslinking solution. The polymeric mixture with the dispersed nanoparticles was left to crosslink for 30 minutes after which the resultant scaffolds were removed and allowed to dry at $25^{\circ} \mathrm{C}$. NaCMC-PEO-ECL crosslinked scaffolds were then agitated in a $1 \% \mathrm{v} / \mathrm{v} \mathrm{HCl}$ solution for 15 minutes. This was undertaken in an attempt to reduce the swelling properties of the scaffold thereby improving erosion and drug release when potentially used intracranially.

\section{Characterization Studies}

Investigation of the chemical structural transitions of the nanoparticles and scaffold

Fourier Transform Infrared (FTIR) spectroscopy was utilized to assess possible structural transitions which had occurred during the preparation process by comparing the functional groups of the parent compounds with that of the formulations produced. Polymer, nanoparticle and scaffold samples were analysed using a Perkin Elmer FTIR Spectrometer (Spectrum 100, Beaconsfield, United Kingdom). Spectra are recorded over the range $4000-625 \mathrm{~cm}^{-1}$, with a resolution of $4 \mathrm{~cm}^{-1}$ and 32 accumulations.

Transmission electron microscopy analysis of the synthesized nanoparticles

Nanoparticles were dispersed in methanol and sonicated to deflocculate the particles. The samples were placed on carbon grids and analysed using transmission electron microscopy (TEM) (JEOL $1200 \mathrm{EX}, 120 \mathrm{keV}$ ) and photomicrographs were obtained to study the morphology of the individual particles produced.

Analysis of the surface morphology of the multipolymeric scaffold

Scanning electron microscopy (SEM), (JEOL, Tokyo, Japan) was employed for observation of the surface morphology of the multipolymeric scaffold. Samples were coated with carbon and gold-palladium for 10 minutes after which they were visualized between $5-9 \mathrm{keV}$ under different magnifications. SEM micrographs were obtained at various magnifications and analyzed for description of the surface morphology. The degree of polymer entanglement, network density and porosity of the scaffold was qualitatively determined using the photomicrographs obtained.

\section{Determination of nanoparticle size and zeta potential}

A ZetaSizer Nano ZS (Malvern Instruments Ltd, UK) was utilized to determine the average size and size distribution of the prepared nanoparticles employing non-invasive back scatter (NIBS) technology with particle size distribution determined using dynamic light scattering. Brownian motion was measured and related to particle size. The Stokes-Einstein equation (Equation 1) was used to determine the translational diffusion coefficient (D), which defined the velocity of Brownian motion. D depends on size of the particle core and the 
surface structures on the particle and the concentration and type of ions in the medium. Particle size was calculated using the translational diffusion coefficient.

$$
\mathrm{d}(\mathrm{H})=k \mathrm{~T} / 3 \pi \eta \mathrm{D} \quad(\text { Equation 1) }
$$

Where: $\mathrm{d}(\mathrm{H})=$ hydrodynamic diameter, $\mathrm{D}=$ translational diffusion coefficient

$\mathrm{k}=$ Boltzmann's constant

$\mathrm{T}=$ absolute temperature

$\eta=$ viscosity

Texture profile analysis to assess the physicomechanical properties of the scaffold Textural profiling of the 3D core of the crosslinked scaffold enabled characterization of the physicomechanical properties of the prepared multipolymeric scaffold. A Texture Analyzer (TA.XTplus, Stable Microsystems, Surrey, UK) was employed to establish various stress-strain parameters of the polymeric scaffold. Samples were assessed in both the hydrated and unhydrated states. Force-Distance and ForceTime profiles were obtained and the Matrix Resilience and Matrix Hardness were computed.

\section{In vitro swelling studies on the multipolymeric scaffold}

The NaCMC-PEO-ECL multipolymeric scaffold was tested for change in volume after exposure to $100 \mathrm{~mL}$ of PBS at predetermined intervals to assess the degree of swelling of the polymer scaffold. Samples were removed from the PBS, blotted on filter paper, after which the mean of three diameters and lengths was calculated following measurements using a callipers while the volume (V) was calculated employing Equation 3.

$$
V=\pi r^{2} l \quad \text { (Equation 2) }
$$

Where, $r$ is the radius $(\mathrm{mm})$ and $l$, the length $(\mathrm{mm})$. Studies were conducted in triplicate to ensure accuracy of the results obtained.

Drug entrapment efficiency studies on the prepared nanoparticles

Drug entrapment efficiency (DEE) was assessed by homogenizing $100 \mathrm{mg}$ of the AZT-loaded nanoparticles in $100 \mathrm{~mL}$ of PBS, pH 7.4 (Polytron PT2000, Kinematika AG, Switzerland) to completely release all entrapped AZT. Free drug molecules were removed from the nanoparticle formulation prior to homoginization by washing the nanoparticles in distilled water. The total content of entrapped AZT was established in triplicate by means of ultraviolet (UV) spectroscopy, (Specord 40, Analytik Jena, AG, Germany) at a wavelength of $267 \mathrm{~nm}$. Due to possible interference of the polymer and excipients used to prepare the samples, a solution of $100 \mathrm{mg}$ of drug-free nanoparticles dissolved in PBS was used as a reference. Equation 4 was employed to calculate the DEE value.

$$
\% D E E=D_{a} / D_{t} \times 100 \quad(\text { Equation 3) }
$$

Where $D_{a}$ is the actual quantity of drug $(\mathrm{mg} / 100 \mathrm{~mL})$ and $D_{t}$ is the theoretical quantity of drug $(\mathrm{mg} / 100 \mathrm{~mL})$ entrapped within the formulation. The study was performed on five samples, with an average DEE being calculated to ensure accuracy of results obtained.

\section{In vitro drug release studies}

Drug release studies were performed on AZTloaded nanoparticle formulations and the multipolymeric scaffold containing the AZTloaded alginate nanoparticles by immersing both sets of formulations in $100 \mathrm{~mL}$ PBS separately and placing them in an orbital shaking incubator $\left(25 \mathrm{rpm}, 37^{\circ} \mathrm{C}\right)$. Samples were withdrawn at predetermined intervals and were analysed using UV spectroscopy at $267 \mathrm{~nm}$ wavelength for AZT concentration.

\section{RESULTS}

\section{Assessment of infrared spectroscopy data for chemical structure transition analysis}

Figure 1 illustrates the FTIR spectra of prepared nanoparticle and scaffold compared with the parent polymeric compounds and AZT. In Figure1b characteristic hydroxyl band between 3600 and $3200 \mathrm{~cm}^{-1}$ of the alginate molecule is observed in the spectra of alginate nanoparticle aswell. Peaks observed at 1592 and $1406 \mathrm{~cm}^{-1}$ are attributed to asymmetric and symmetric - $\mathrm{COO}$ stretching. In the FTIR spectra of pectin (Figure $1 b$ ), the presence of hydroxyl groups was also observed between 3600 and $3200 \mathrm{~cm}^{-1}$ and -COOstretching occurred at $1590 \mathrm{~cm}^{-1}$. The peak at $3458 \mathrm{~cm}^{-1}$ in the AZT spectra is indicative of hydroxyl substituents and the bands between 3200 and $3000 \mathrm{~cm}^{-1}$ are due to amine groups. Bands between 2100 and $1900 \mathrm{~cm}^{-1}$ are attributed to multiple bonded $\mathrm{N}_{2}$ molecules. The vibration energies of $-\mathrm{C}=\mathrm{O}$ moieties present in the AZT, molecule was observed at 2813 and $1671 \mathrm{~cm}^{-1}$. Characteristic bands were present in the spectra of PVA at 3274 and $2907 \mathrm{~cm}^{-1}$ indicating the presence of hydroxyl groups and $-\mathrm{C}-\mathrm{H}$ stretching vibrations respectively. The bands present 
between 1300 and $1000 \mathrm{~cm}^{-1}$ are due to the bending vibrations of alcohol moieties present in PVA molecule or to $-\mathrm{C}-\mathrm{O}$ stretching vibrations (Figure 1b). The alginate nanoparticle formulation presented with bands between 3600 and $3200 \mathrm{~cm}^{-1}$ indicating the presence of possible hydroxyl or amino substituents (Figure 1b). The FTIR spectra of pectin nanoparticles (Figure 1c) displayed a broad band between 3600 and $3200 \mathrm{~cm}^{-1}$ indicating the presence of hydroxyls groups. A peak at $2944 \mathrm{~cm}^{-1}$, attributed to $-\mathrm{C}-\mathrm{H}$ stretching vibrations observed on the spectra of PVA and pectin is absent in nanoparticle formulation.

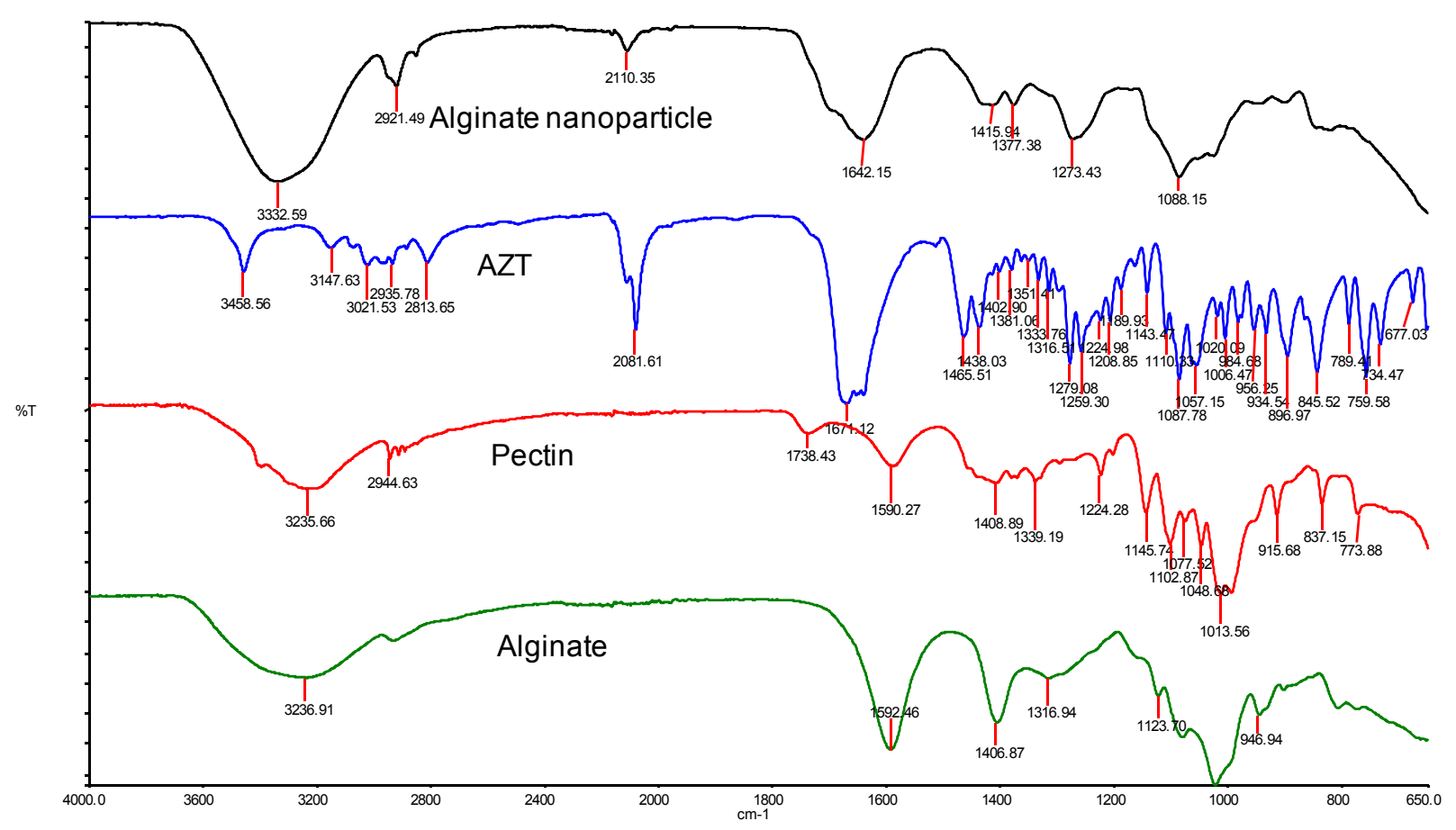

(a)

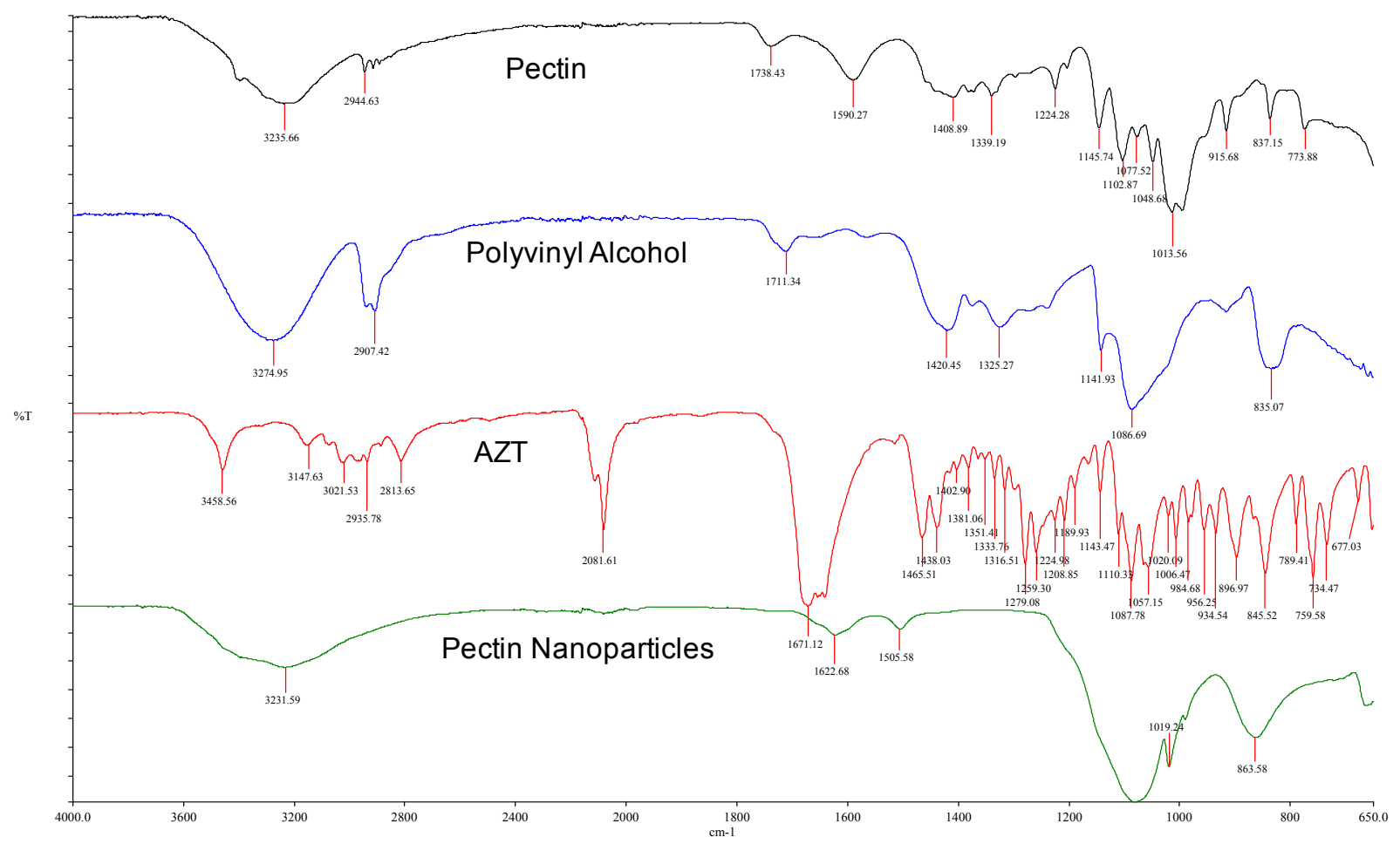

(b) 


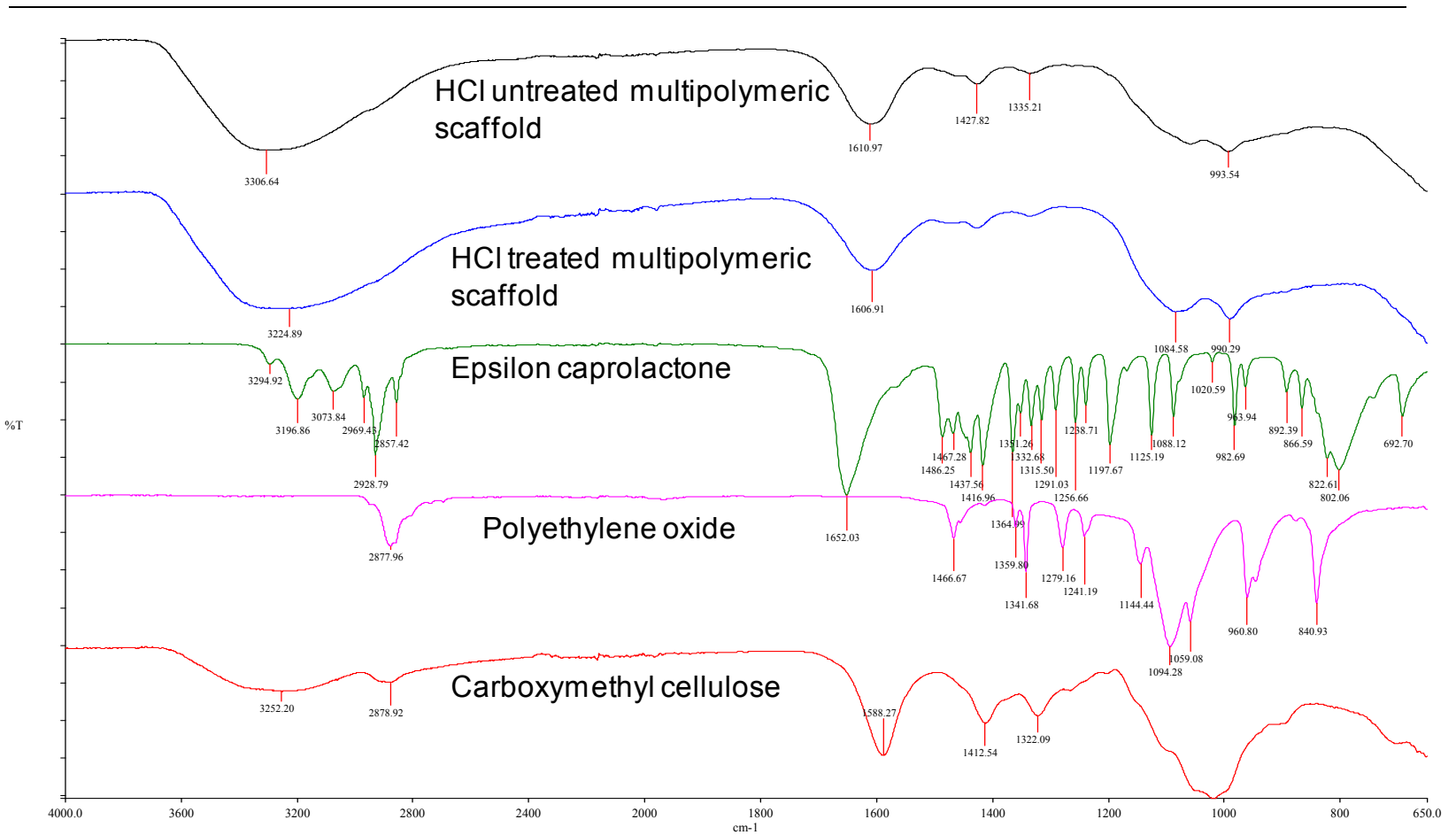

(c)

Figure 1. (a) FTIR spectra of nanoparticles prepared by means of a controlled gelification of alginate compared to the parent compounds; (b) FTIR spectra of nanoparticles prepared using the salting out approach; (c) FTIR of the prepared multipolymeric scaffold compared to the parent polymer compounds.

The FTIR spectra in Figure 1d illustrate the parent polymer compounds compared with the prepared multipolymeric scaffold treated and untreated with $\mathrm{HCl}$. From the FTIR spectra of NaCMC, a broad band at $3252 \mathrm{~cm}^{-1}$ can be observed due to hydroxyl stretch vibrations and bands at 2878 and $1322 \mathrm{~cm}^{-1}$ are due to $-\mathrm{C}-\mathrm{H}$ groups. The peak at $1588 \mathrm{~cm}^{-1}$ is typical of the carboxymethyl ether, ($\mathrm{CH}_{2}-\mathrm{COOH}$ ), and that at $1412 \mathrm{~cm}^{-1}$ is due to symmetric stretching of the carboxylate group. Bands present at $1018 \mathrm{~cm}^{-1}$ are representative of $>\mathrm{CH}-\mathrm{O}-\mathrm{CH}_{2}$ stretching. Bands present in the FTIR spectra of PEO at $960 \mathrm{~cm}^{-1}$ and between 900 and $1250 \mathrm{~cm}^{-1}$ are due to $-\mathrm{C}-\mathrm{O}$ group vibrations and $-\mathrm{C}-\mathrm{O}-\mathrm{C}$ stretching respectively. Those at $2877 \mathrm{~cm}^{-1}$ and between 1400 and $1300 \mathrm{~cm}^{-1}$ are due to $-\mathrm{C}-\mathrm{H}$ stretching and the band at $840 \mathrm{~cm}^{-1}$ is due to $-\mathrm{C}-\mathrm{H}$ bending vibrations. Bands between 1060 and $1150 \mathrm{~cm}^{-1}$ can be attributed to the aliphatic ether moieties in the structure of PEO. The ECL spectra displays characteristic peaks between 1050 and $1300 \mathrm{~cm}^{-1}$, indicative of the cyclic ester present in the structure of ECL (Figure 1a) and the carbonyl $-\mathrm{C}=\mathrm{O}$ stretching was observed at $1652 \mathrm{~cm}^{-1}$ and between 3400 and $3150 \mathrm{~cm}^{-1}$. Bands between 2800 and $3000 \mathrm{~cm}^{-1}$ are due to $-\mathrm{C}-\mathrm{H}$ stretching.
Transmission electron microscopy image analysis of the nanoparticles

TEM conducted on the drug-loaded alginate nanoparticles, prepared by means of the controlled gelification of alginate approach, revealed the presence of spherical particles, whereas drug-free nanoparticles displayed a tendency to agglomerate, as seen in Figure 2a-b. Micrographs obtained from drug-free $\mathrm{Zn}$-pectin nanoparticles, prepared using the salting out approach, also revealed agglomeration, and the drug-loaded Zn-pectin nanoparticles showed spherical morphology as observed in Figure 2c-d.

\section{Surface morphological analysis of the multipolymeric scaffold}

SEM micrographs of NaCMC-PEO-ECL multipolymeric scaffolds revealed a porous polymer matrix, as observed in Figure 3. Scaffolds exposed to $1 \% \mathrm{v} / \mathrm{v} \mathrm{HCl}$ exhibited smaller, more uniform pores than scaffolds untreated with $\mathrm{HCl}$ (Figure 3(a)-(b)). $\mathrm{HCl}$ treated scaffolds contained large areas of densely packed polymer matrices, interspersed by porous regions (Figure 3b), whereas $\mathrm{HCl}$ untreated scaffolds contained immense craters within the matrix and densely packed polymer regions were rarely noted on the photomicrographs (Figure 3a). SEM was 
also conducted on multipolymeric scaffolds crosslinked only once. Photomicrographs obtained for these samples contained larger pores and more craters within the polymer matrix unlike smaller pores present in multi-crosslinked scaffolds. Air pockets are clearly visible in the scaffold matrix as seen in Figure 3(c)-(d).

\section{Nanoparticle size and zeta potential}

Zeta potential and particle size distribution was determined in deionised water. Zn-Pectin nanoparticles displayed an average size distribution of $1323 \mathrm{~nm} \quad(\mathrm{SD}<0.008)$, with a polydispersity index (PDI) of 0.816 for drug-free nanoparticles and $119.6 \mathrm{~nm} \quad(\mathrm{SD}<0.005)$, PDI 0.676, for AZT-loaded nanoparticles (Figure 4). Multiple wide peaks were obtained (Figure 4a) and microparticles observed with drug free nanoparticles as agglomerates were formed which could not be redispersed upon sonication of the nanoparticles prior to analysis (Figure 5).

Drug-free pectin nanoparticles displayed an average zeta potential of $-0.012 \mathrm{mV}(\mathrm{SD}<0.001)$, whereas AZT-loaded nanoparticles displayed an average zeta potential of $-0.419 \mathrm{mV}(\mathrm{SD}<0.004)$.
The average zeta potential of AZT-loaded alginate nanoparticles was $-6.39 \mathrm{mV}(\mathrm{SD}<0.003)$ and that of drug-free nanoparticles was $-3.53 \mathrm{mV}$ $(\mathrm{SD}<0.002)$ (Figure 6).

\section{Texture profile analysis}

Texture analysis performed on unhydrated samples revealed a decrease in the resilience of the $\mathrm{HCl}$ treated matrix compared to untreated systems with a matrix resilience (MR) of $12.83 \%$ $(\mathrm{SD}<0.004)$ as compared $18.12 \% \quad(\mathrm{SD}<0.004)$ obtained with untreated scaffold. However, hardness of both treated and untreated matrices was similar, with treated scaffold having a mean value of $3.45 \mathrm{~N} / \mathrm{mm}(\mathrm{SD}<0.001)$ and untreated scaffold having a mean value of $3.712 \mathrm{~N} / \mathrm{mm}$ $(\mathrm{SD}<0.006)$ (Figure 7).

A decline in matrix resilience was accounted upon scaffold hydration, both in the case of $\mathrm{HCl}$ treated and untreated samples, the scaffolds unexposed to $\mathrm{HCl}$ displaying a mean resilience of $8.043 \%(\mathrm{SD}<0.004)$ and scaffolds exposed to $\mathrm{HCl}$ displaying a mean resilience of $6.361 \%$ $(\mathrm{SD}<0.004)$ (Figure 8).

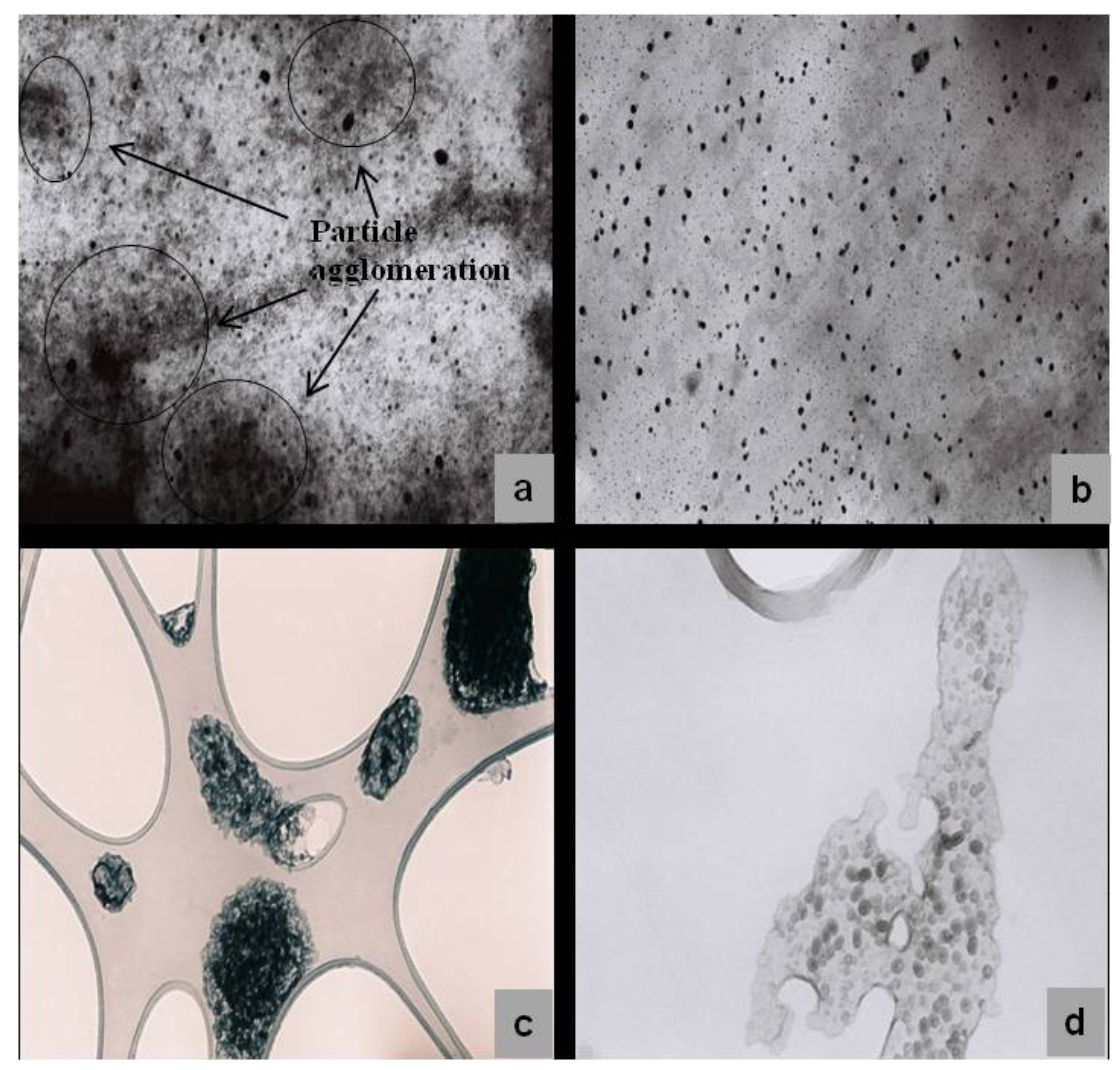

Figure 2. TEM images of nanoparticles: (a) Drug free alginate nanoparticles displaying agglomeration, $(1 \mathrm{~mm}=20 \mathrm{~nm})$, (b) AZT loaded alginate nanoparticles displaying the presence of spherical particles, $(1 \mathrm{~mm}=20 \mathrm{~nm})$, (c) Drug free $\mathrm{Zn}$ pectin nanoparticles displaying agglomeration, (d) AZT loaded Zn-pectin nanoparticles displaying inter-dispersed spherical structures in the absence of agglomeration, $(1 \mathrm{~mm}=10 \mathrm{~nm})$. 


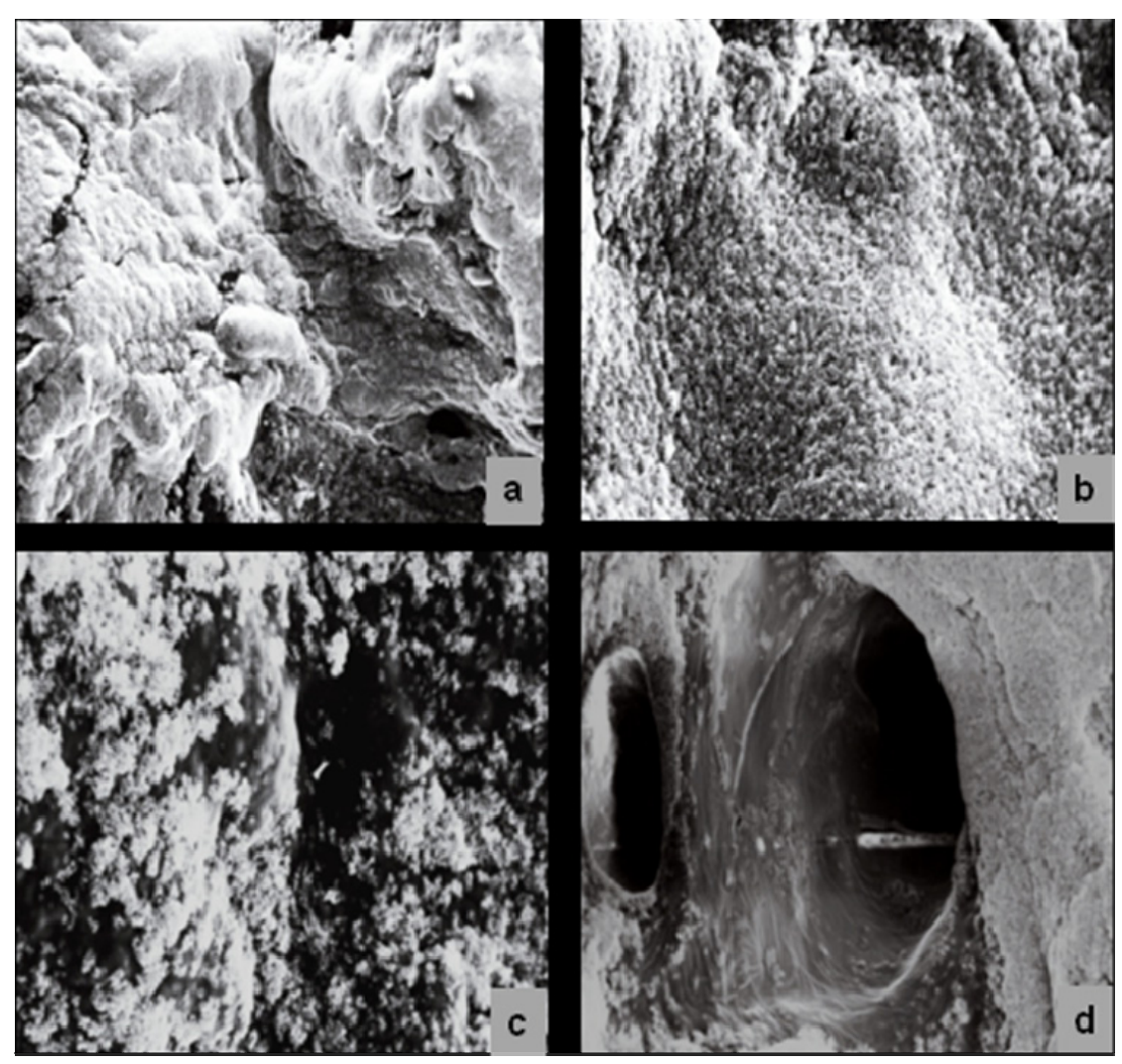

Figure 3. Scanning Electron Micrograph of (a)-(b) multicrosslinked polymeric scaffolds: (a) HCl untreated, displaying densely packed polymer matrix due to efficient crosslinking of the multipolymeric scaffold, $(1 \mathrm{~mm}=50 \mu \mathrm{m})$. (b) $\mathrm{HCl}$ treated, displaying uniform pores present within the polymer matrix of scaffolds which can efficiently entrap drugloaded nanoparticles, thereby serving to modulate drug release $(1 \mathrm{~mm}=125 \mu \mathrm{m})$. (c)-(d) polymeric scaffolds crosslinked once: (c) Craters can be seen between the pores in the matrix due to air bubbles in the polymer solution during scaffold preparation $(1 \mathrm{~mm}=50 \mu \mathrm{m})$. (d) Upon closer magnification, densely packed polymer regions and porous areas can be observed surrounding the craters $(1 \mathrm{~mm}=250 \mu \mathrm{m})$.

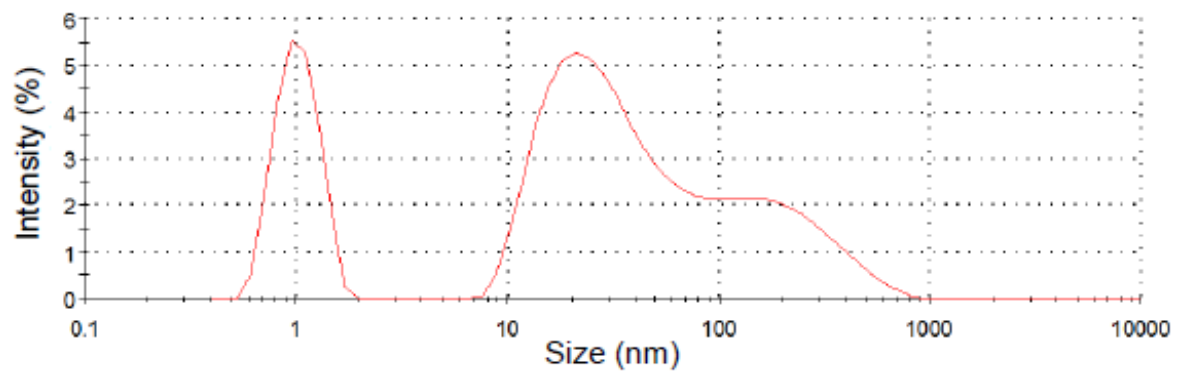

(a)

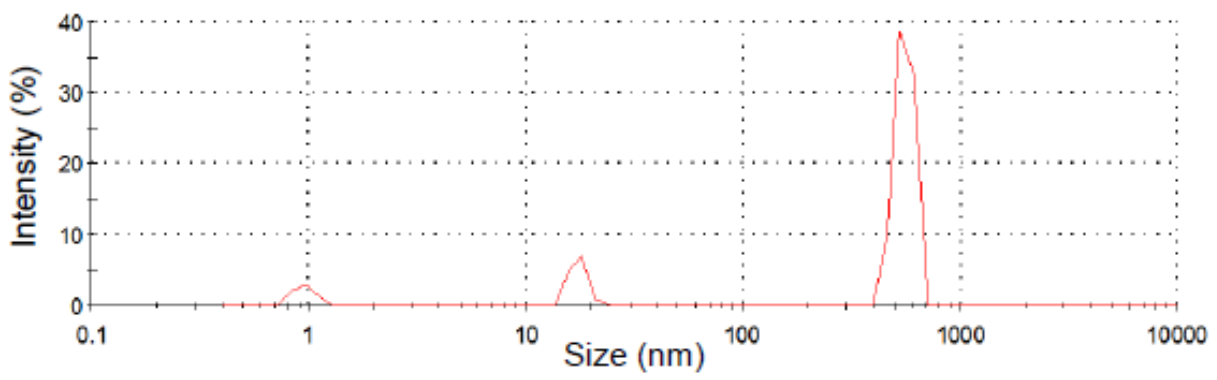

(b)

Figure 4. Size distribution profile of Zn-Pectin nanoparticles (a) drug-free nanoparticles, (n=3) (b) AZT-loaded nanoparticles, $(n=3)$. 


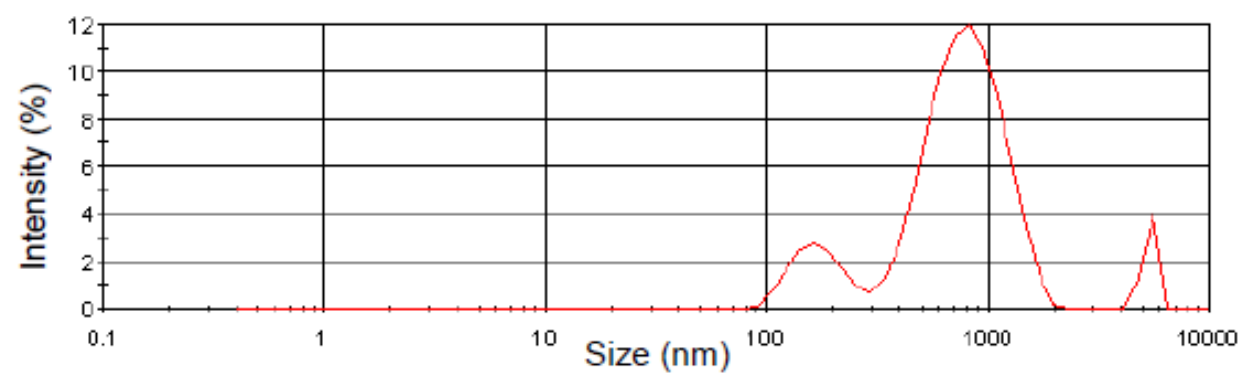

(a)

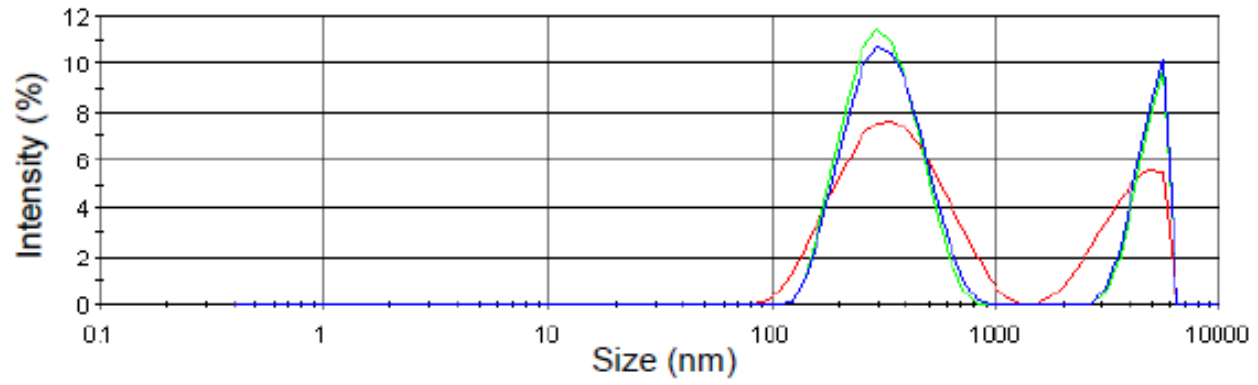

(b)

Figure 5. Size distribution profile of alginate nanoparticles: (a) drug-free nanoparticles, (n=3) (b) AZT-loaded nanoparticles, $(\mathrm{n}=3)$.

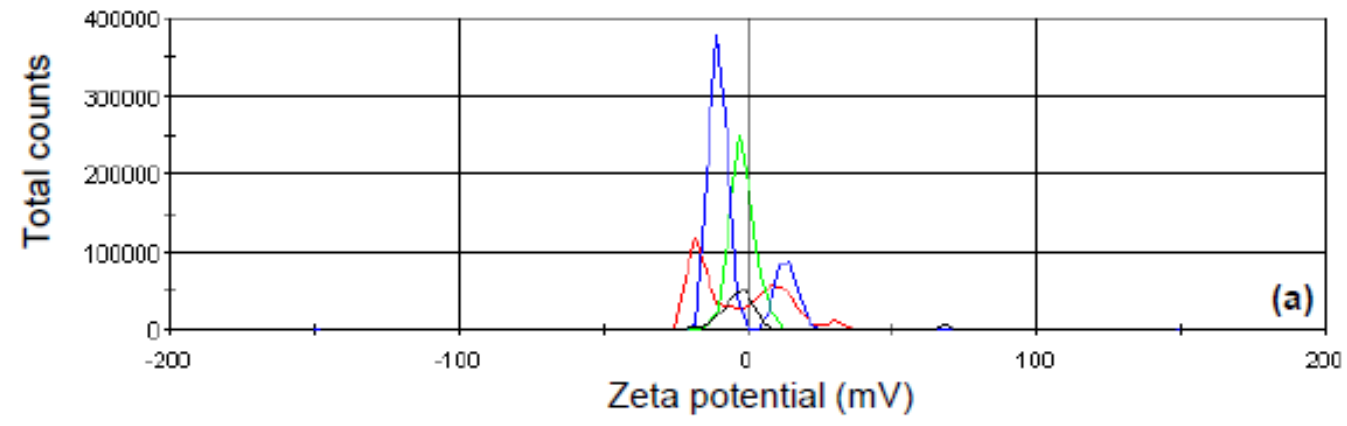

Figure 6. Zeta potential profile of AZT-loaded alginate nanoparticles, $(\mathrm{n}=3)$.

\section{Matrix swelling analysis}

The polymeric matrix exposed to $\mathrm{HCl}$ treatment exhibited reduced swelling behaviour. However, both sets of scaffolds still exhibit appreciable swelling behaviour as seen in Figure 9. A reduced erosion of the multipolymeric scaffolds treated with $\mathrm{HCl}$, was noticed in the first 24hours as compared to untreated scaffolds (Figure 9). However, upon prolonged exposure to PBS, $\mathrm{pH}$ 7.4, NaCMC-PEO-ECL multipolymeric scaffolds, treated and untreated with $\mathrm{HCl}$, displayed a weight gain. Multipolymeric scaffolds exposed to $\mathrm{HCl}$ displayed a larger percentage weight gain than those unexposed to $\mathrm{HCl}$.

\section{Drug entrapment efficiency and in vitro zidovudine release studies}

A calibration curve was generated for AZT using PBS, pH 7.4 at $25^{\circ} \mathrm{C}$, employing a UV spectrophotometry at the wavelength of maximum absorption, $267 \mathrm{~nm}$. Alginate nanoparticles exhibited superior DEE, $91.1 \%$, as compared to $76.53 \%$ obtained with Zn-pectin nanoparticles.

Biphasic release was observed with both nanoparticle formulations, consisting of an initial burst release of drug within hours of exposure to PBS, followed by a constant release rate of AZT over the remaining 30days of nanoparticle analysis (Figure 10a). Zn-pectin and alginate nanoparticles displayed a burst release of 7.9\% and $14.9 \%$ respectively of the entrapped drug after 1 hour post exposure to PBS. Incorporation of alginate nanoparticles, chosen due to its superior DEE, into the polymeric scaffold significantly retarded drug release, $2.341 \%$ $(\mathrm{SD}<0.005)$ obtained with NaCMC-PEO-ECL scaffolds untreated with $\mathrm{HCl}$ and $1.576 \%$ $(\mathrm{SD}<0.005)$ obtained with NaCMC-PEO-ECL scaffolds treated with $\mathrm{HCl}, 30$ days post exposure to PBS, pH 7.4 (Figure 10b). 

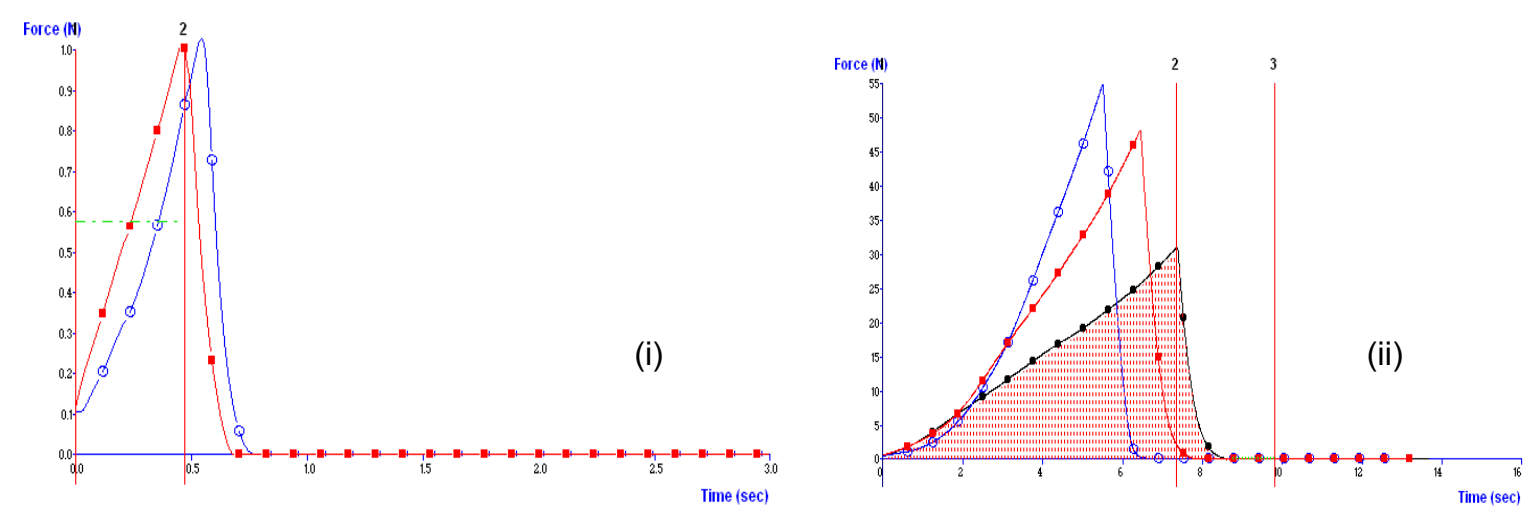

(a)
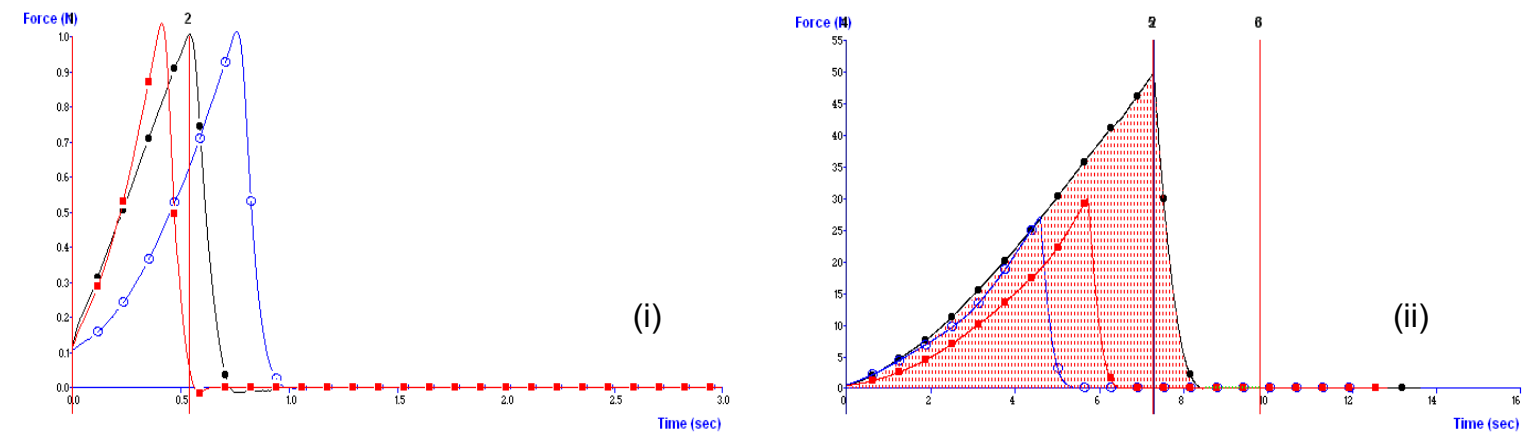

(b)

Figure 7. Texture analysis: (a) Multipolymeric scaffold unexposed to $\mathrm{HCl}$ (i) hardness and (ii) resilience profiles; (b) Multipolymeric scaffold exposed to $\mathrm{HCl}$ (i) hardness and (ii) resilience profiles, $(\mathrm{n}=3)$.

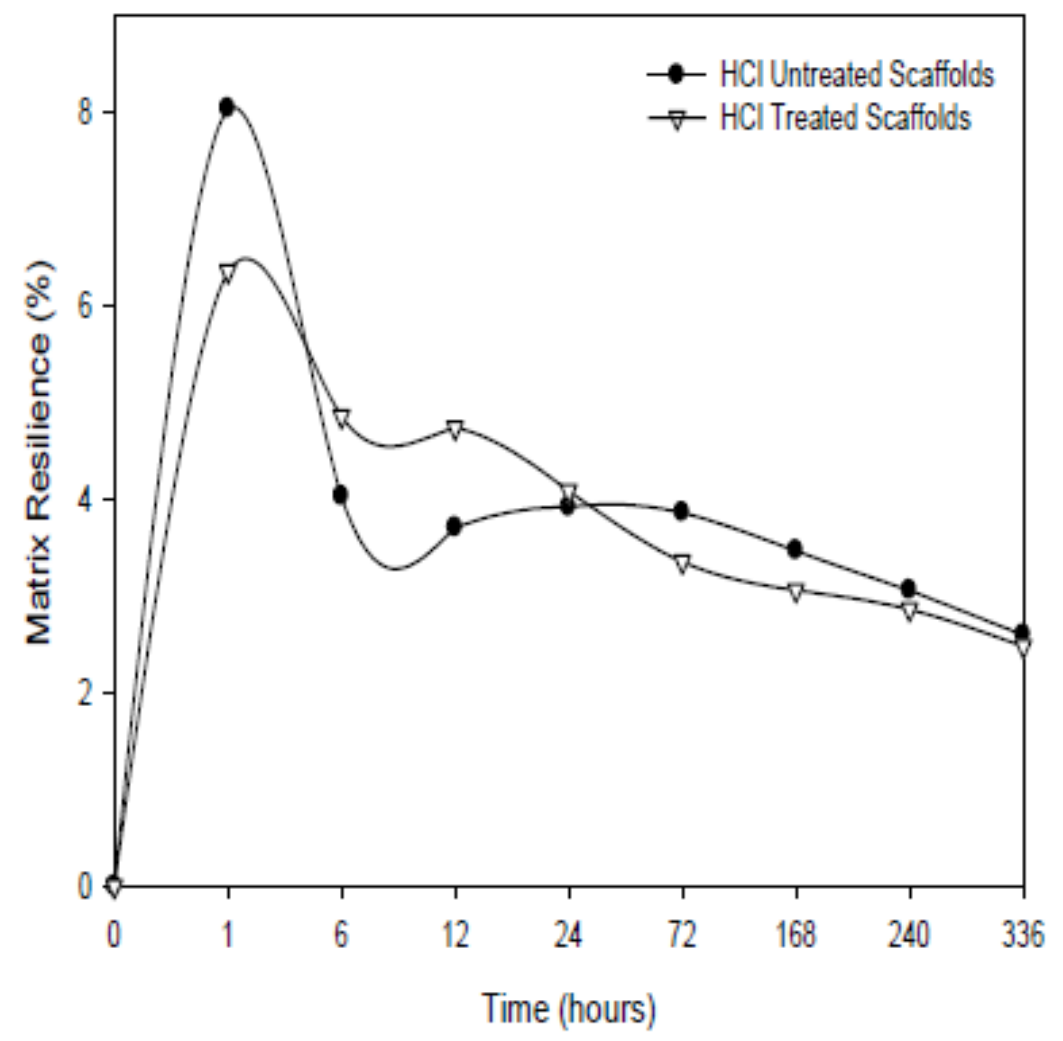

Figure 8. Percentage resilience of $\mathrm{HCl}$ treated and untreated scaffolds, upon exposure to $\mathrm{PBS}, \mathrm{pH} 7.4(\mathrm{n}=3, \mathrm{SD}<0.004)$ 


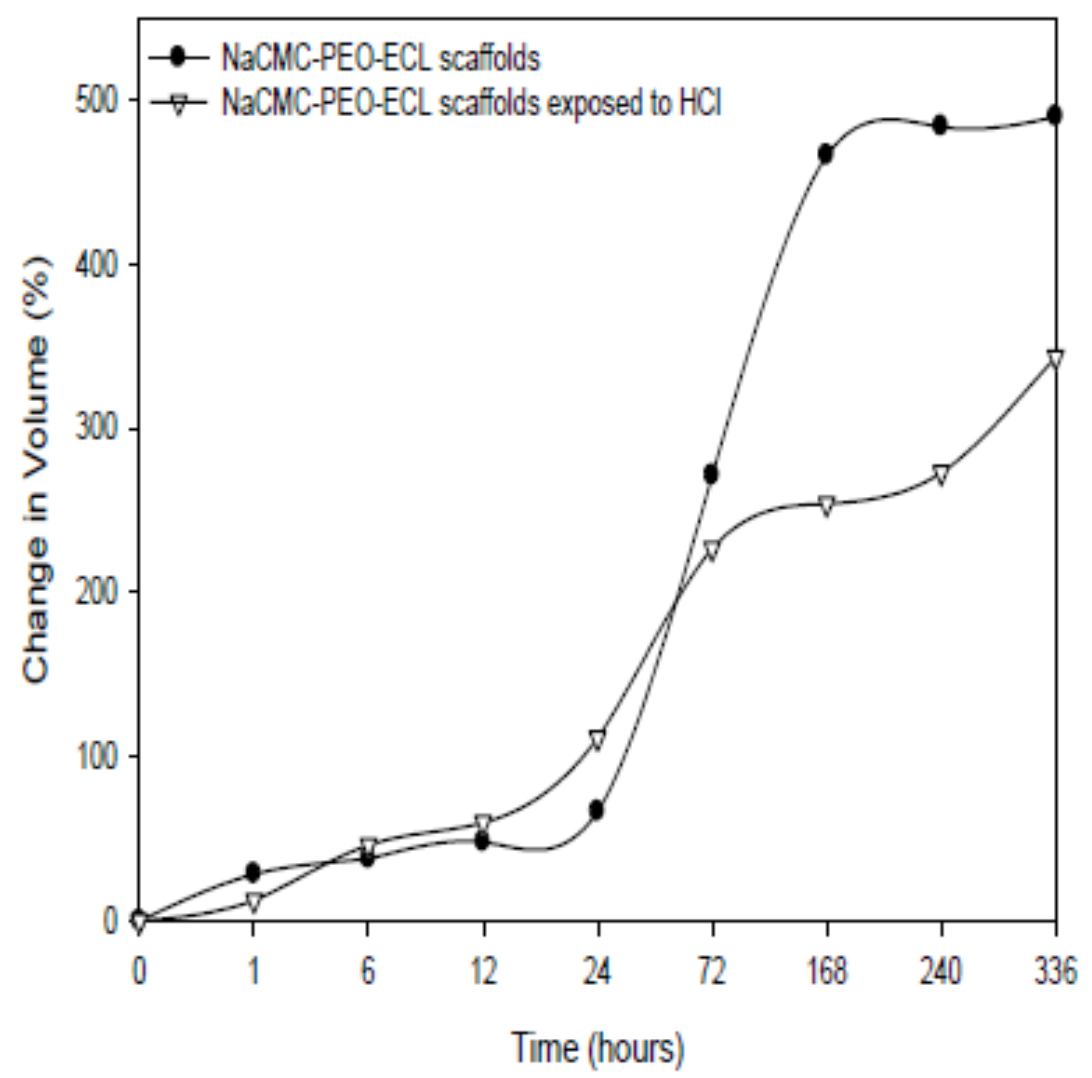

(a)

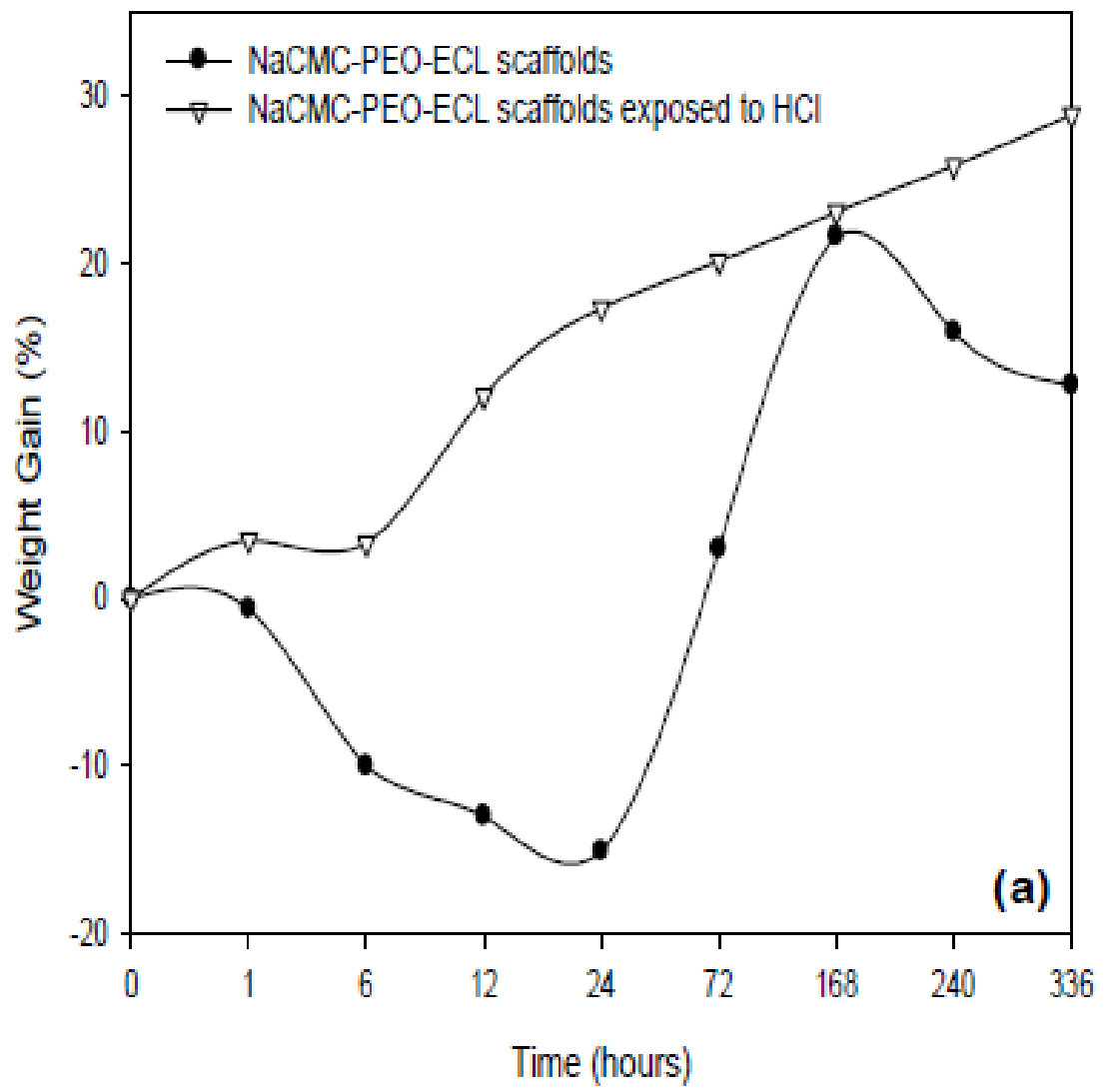

(b)

Figure 9. Swelling behavior of $\mathrm{HCl}$ treated and untreated multipolymeric scaffolds (a) Volume change, (b) Percentage weight change, $(n=3)$. 


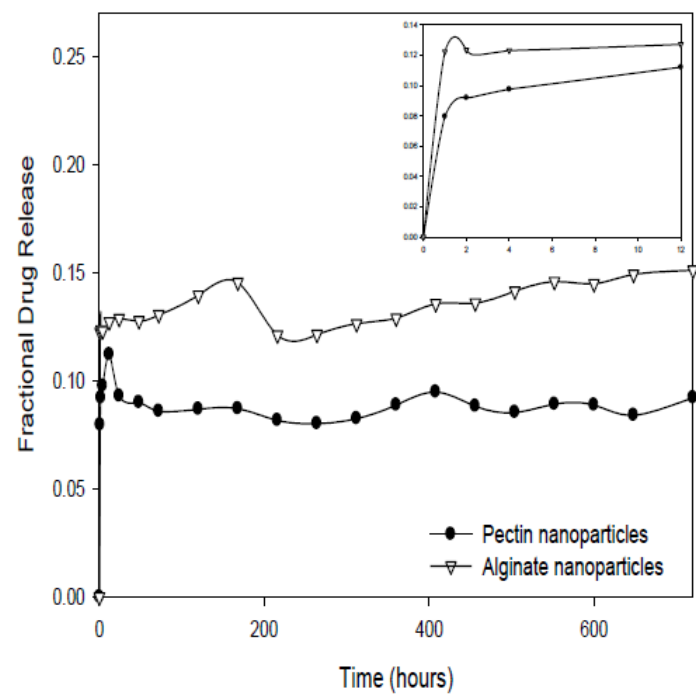

(a)

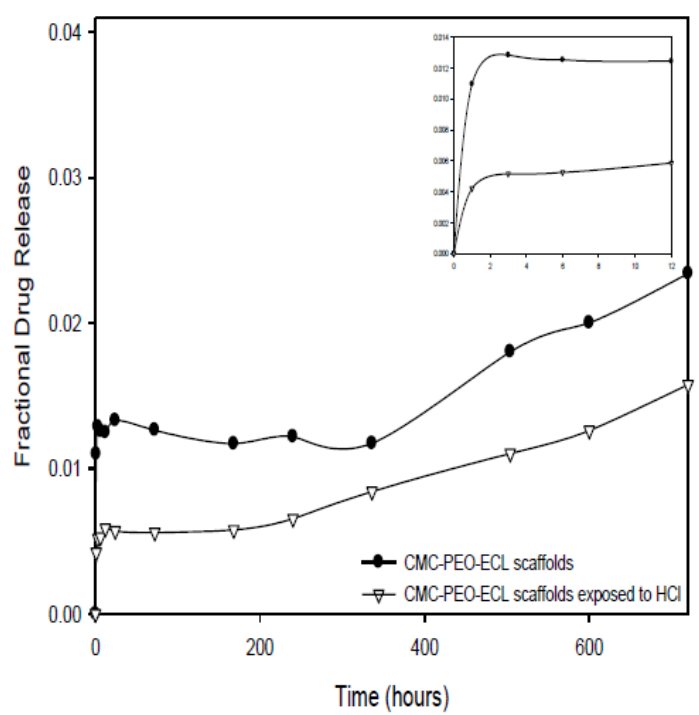

(b)

Figure 10. Drug release profile of (a) Zn-pectin and alginate nanoparticles and (b) alginate nanoparticles dispersed within $\mathrm{HCl}$ treated and untreated multipolymeric scaffold, under cerebrospinal fluid simulated conditions (20rpm, $37^{\circ} \mathrm{C}, 0.1 \mathrm{M}$ PBS, $\left.\mathrm{pH} 7.4\right)(\mathrm{n}=3 ; \mathrm{SD}<0.005$ in all cases $)$. Insets show release from free nanoparticles.

\section{DISCUSSION}

The analysis of prepared nanoparticles through FTIR studies indicated that the basic polymeric structures of the parent compounds was maintained, however surface interactions occurred in the formation of both Zn-pectin and alginate nanoparticles and the scaffold, which is represented by the shifting of the transmittance peaks in the nanoparticle and the multipolymeric scaffold preparations. In prepared alginate nanoparticles the band at $3332 \mathrm{~cm}^{-1}$ is more defined than that of alginate, indicating possible interactions of the polymers with drug AZT, as AZT has an amino substituent present at $3458 \mathrm{~cm}^{-1}$ as observed in the FTIR spectra. Polymer interactions with AZT are further supported by the presence of a band at $2110 \mathrm{~cm}^{-1}$ which can be attributed to $\mathrm{N}_{2}$ substitution or bonding (e.g. ionic bonds). Shifting of the $-\mathrm{COO}$ - stretch to $1642 \mathrm{~cm}^{-1}$ in the nanoparticle spectra indicates possible interaction of the polymers with AZT or $\mathrm{CaCl}_{2}$. The presence of hydroxyl band in pectin nanoparticles is consistent with the spectra of the parent polymers, PVA and pectin. The $-\mathrm{C}-\mathrm{H}$ stretching vibration in PVA and pectin is found absent in the formed nanoparticle spectra supporting interaction between the formulation constituents. The carboxymethyl ether groups of $\mathrm{NaCMC}$ can be observed in both the multipolymeric scaffolds exposed and unexposed to $\mathrm{HCl}$ at 1610 and $1606 \mathrm{~cm}^{-1}$, respectively and bands present between 3450 and $3200 \mathrm{~cm}^{-1}$ are due to hydroxyl groups. The $\mathrm{C}-\mathrm{O}$ groups present in the polymer structure allows for complexation of PEO with other polymers and salts $(33,34)$. A shift in the occurrence of the hydroxyl groups can be seen from 3306 to $3224 \mathrm{~cm}^{-1}$ in the untreated and $\mathrm{HCl}$ treated scaffolds respectively, indicating possible interactions of the hydroxyl groups in $\mathrm{NaCMC}$ with $\mathrm{H}^{+}$ions upon exposure to $\mathrm{HCl}$. This is further supported by fewer bands present in the FTIR spectra of $\mathrm{HCl}$ treated multipolymeric scaffolds as compared to untreated scaffolds.

The micrographs of nanoparticles prepared using both the salting-out and the controlled gelification of alginate approach revealed agglomeration in case of the drug-free nanoparticles, whereas the drug-loaded formulations appeared more disperse. This observation relates to the low charge distribution within the drug-free colloidal system. The addition of AZT influenced the charge distribution by interacting with the polymer as confirmed by the FTIR data. This was further supported by the zeta potential analysis.

The existence of craters in micrographs of prepared scaffolds unexposed to $\mathrm{HCl}$ can be explained on account of the two polymers used for the preparation of the multipolymeric scaffold being hydrogels, namely $\mathrm{NaCMC}$ and PEO. Hydrogels, which are three-dimensional crosslinked polymer capable of extreme swelling due to absorption and retention of water, have a loosely crosslinked structure due to their hydrophilic nature, resulting in the presence of air 
bubbles in the scaffolds unexposed to $\mathrm{HCl}$ (35). Exposure of the scaffolds to $\mathrm{HCl}$ causes the COONa group in CMC to change to the acidic $\mathrm{COOH}$ which forms a more compact polymeric matrix (36). The appearance of still larger pores in scaffolds crosslinked only once can be explained by the presence of higher concentrations of $\mathrm{Ca}^{2+}$ and $\mathrm{Al}^{3+}$ ions that form intramolecular complexes within $\mathrm{NaCMC}$, resulting in a tightly coiled polymer matrix in multi-crosslinked scaffolds. Such formulations with large pore size are not ideal for nanoparticle dispersion, supporting the need for multiple crosslinking to achieve an optimum scaffold structure. The distinct bright white areas visible on the multipolymeric scaffolds are due to a high electric charge on the surface accredited to the presence of multivalent ions, present on the surface of the polymer matrix (37), as well as free $\mathrm{H}^{+}$and $\mathrm{Cl}^{-}$ions on scaffolds exposed to $1 \% \mathrm{v} / \mathrm{v} \mathrm{HCl}$. A porous polymer matrix is considered ideal for the dispersion of drugloaded nanoparticles. The drug release is anticipated to enhance as the nanoparticles will be released from polymer matrix when it swells or erodes, allowing for the prolonged delivery of drug. Additionally, premature drug degradation will be prevented and drug toxicity reduced as controlled drug release will be achieved $(4,17)$.

Agglomeration of drug-free nanoparticles both Zn-pectin and alginate - as indicated by TEM micrographs, nanoparticle size zeta potential, can be attributed to poor crosslinking and a low charge distribution within the colloidal systems. Pectin microparticles observed are due to nanoparticle agglomeration and poor crosslinking of pectin with $\mathrm{ZnSO}_{4}$. The large particle size and even the presence of microparticles obtained for drug free nanoparticles, indicated the tendency of the colloidal system to agglomerate and was confirmed with zeta potential analysis. The use of DLS for the analysis of a sample containing a mixture of extremely large and small particles yields large $\mathrm{Z}$-averages as the contribution to the total light scattered by the small particles will be extremely small, Wider peaks in the graph are due to the tendency of nanoparticles to agglomerate, which can be noted by the peak in the $900 \mathrm{~nm}$ range or close to the $1000 \mathrm{~nm}$ in Figure 5a. Agglomeration of the nanoparticles can be reduced by the addition of a surface active agent to the formulation, which will be explored in further studies. The overall poor zeta potential obtained can be attributed to the strong positive surface charge from the $\mathrm{Ca}^{2+}$ ions being shielded due to ionotropic crosslinking of the $\mathrm{Ca}^{2+}$ ions with the carboxyl groups of the guluronic acid residues, which makes up alginate, in which $\mathrm{Ca}^{2+}$ is held in the centre of the 3-dimensional structure. Alginate is an anionic polymer, resulting in the negative zeta potential obtained $(13,38,39)$. Although AZT in an uncharged molecule (40), there is a possible interaction of AZT with the polymers, as observed from the FTIR spectra, with resultant inferior crosslinking of $\mathrm{Ca}^{2+}$ with alginate. Alginate nanoparticles prepared by crosslinking with $\mathrm{CaCl}_{2}$ exhibited superior size distribution compared to the $\mathrm{Zn}$ pectin nanoparticles.

Assessment of MR provides information regarding in vitro degradation and drug release behaviour of the multipolymeric scaffold device. MR also has implications for device storage. It is therefore imperative to have a rigid polymeric scaffold which can withstand fracture during storage and handling of the device. A decline in the resilience of $\mathrm{HCl}$ treated matrix as compared to untreated matrix was expected as $\mathrm{HCl}$ exposed scaffolds presented with less porous matrices due to acid transition of the COONa groups in $\mathrm{CMC}$ to $\mathrm{COOH}$. This could possibly result in a brittle scaffold, accounting for the low MR obtained. Less water will therefore permeate into the polymer matrix leading to reduced disentanglement of the polymer matrix, allowing for prolonged drug release. MR of both sets of scaffold samples declines further with exposure to PBS due to swelling and chain relaxation of the polymer matrix. The viscoelastic property of the scaffold resultantly increases and matrix resilience declines.

Upon treatment with $1 \% \mathrm{v} / \mathrm{v} \mathrm{HCl}$, the carboxylate $\mathrm{COONa}$ groups in $\mathrm{NaCMC}$ are converted to the acidic $\mathrm{COOH}$ form. This $\mathrm{COOH}$ form is less susceptible to ionisation in PBS, as the COO- group has a greater affinity for the $\mathrm{H}^{+}$ ion resulting in a lower degree of ion exchange of the $\mathrm{Na}^{+}$and $\mathrm{H}^{+}$to reform COONa. The polymeric matrix resultantly imbibes less water, reducing the swelling behaviour of $\mathrm{HCl}$ treated scaffold. Exposure to $\mathrm{HCl}$ also leads to a more densely crosslinked polymer matrix which is less prone to swelling than the loosely packed untreated scaffolds. Exposure to $\mathrm{HCl}$ also leads to a more densely crosslinked polymer matrix which is less prone to swelling than the loosely packed untreated scaffolds. Still, an appreciable swelling in both scaffold types could be attributed to the highly hydrophilic nature of the constituent polymers which enhances the rate of uptake and water uptake capacity. A comparative decrease in the amount of water penetrating into the $\mathrm{HCl}$ treated scaffold reduces the disentanglement of this polymeric matrix, attributing to the reduced erosion observed for this scaffold compared to 
$\mathrm{HCl}$ untreated scaffold, in first 24hours of PBS exposure. However, the weight gain in both types of scaffold on prolonged exposure to PBS is thought to be due to sequestration of phosphate and $\mathrm{Na}^{+}$ions indicating degree of entrapped moisture and ions within of the polymer matrix accounting for the gain in weight of the scaffolds and resultant decrease of the entanglement of the polymer chains (36).

The comparatively high DEE recorded for the alginate nanoparticle formulation is accredited to the ability of alginate to form a gel in the presence of an aqueous medium, with the hydrophilic AZT entrapped within this gel structure along with the nanoparticle precipitation upon crosslinking of the alginate-AZT solution with $\mathrm{CaCl}_{2}(16,17,41)$. The high DEE of alginate nanoparticles, compared to the pectin nanoparticles, coupled to their even size distribution with a comparatively stable formulation qualified them for further analysis. Drug release obtained with alginate nanoparticles was more suitable than that of pectin nanoparticles for dispersion within a multipolymeric scaffold matrix for possible application as an implantable drug delivery device. Dispersing the alginate nanoparticles in multipolymeric scaffold was aimed to prolong the drug release. However, the burst release of drug from both the types of nanoparticle was attributed to association of the AZT onto the surface of the polymers by weak interactive forces, which was reversed upon exposure to PBS. The nanopartcle liberation and subsequent drug release from polymeric scaffold is governed primarily by two factors: scaffold swelling and erosion. Erosion of the scaffold is due to weakening of the crosslinked bonds with exposure to PBS (pH 7.4). ECL present in the matrix degrades by means of hydrolysis of the ester bonds, without creating a localised acidic environment upon degradation. When exposed to the $\mathrm{CaCl}_{2}$ salt solution, $\mathrm{CaCl}_{2}$ either forms bonds with the ester groups on the lactone ring or the $\mathrm{Ca}^{2+}$ surrounds the ester group and protects it from degradation $(26,28,31)$. PEO degrades by means of hydrolysis of the ester bonds. CMC and PEO imbibes large quantities of water due to their hydrophilic nature, resulting in rapid degradation of $\mathrm{PEO}$, subsequently causing break down of the polymer scaffold. The degree of swelling and subsequent drug release is determined by the availability of hydrogen bonds in the polymer matrix for water molecules to attach to. For scaffolds treated with $1 \% \mathrm{v} / \mathrm{v} \mathrm{HCl}$, the availability of such bonds is decreased, thereby resulting in reduced drug release. The PBS penetrated the scaffold matrix, which has small uniform pores as well as air pockets, causing it to erode, allowing for nanoparticles to diffuse out. Nanoparticles released drug by a similar method, with swelling of the nanoparticles upon exposure to PBS playing a major role in drug release. This is consistent with the polymers used to prepare the nanoparticles, being alginate and pectin, which are hygroscopic in nature and prone to swelling in the presence of water. This swelling behaviour would allow for weakening of the crosslinked bonds between the polymers and the $\mathrm{CaCl}_{2}$, and release of drug from the nanoparticles. Swelling of the nanoparticles further facilitates surface erosion and subsequent release of drug $(38,39)$.

\section{CONCLUSIONS}

From the studies conducted, the controlled gelification of alginate method of producing nanoparticles was chosen for further analysis as alginate nanoparticles exhibited a more stable formulation, as determined by zeta potential analysis and smaller particles are formed using this approach. Pectin nanoparticles demonstrated suitable size and drug release properties for application as a drug carrier in the absence of a scaffold matrix. However, further investigations are required to enhance the stability of the formulation and to decrease agglomeration. Dispersing the polymeric nanoparticles within a multipolymeric scaffold served to noticeably decrease the drug release rate. Exposing the prepared multipolymeric scaffolds to $1 \% \mathrm{v} / \mathrm{v} \mathrm{HCl}$ served to reduce the swelling behaviour of the scaffolds, thereby retarding drug release and creating a suitable device for the prolonged release of drug over a period of one month. The nanoparticle-loaded system can be used for the drug delivery, where controlled drug delivery over extended periods is required. A possible application for such a device is as subcutaneous implant to achieve systemic drug release which bypasses the gastrointestinal tract and the liver. This is useful for drugs which are sensitive to the acidic environment of the stomach and susceptible to first pass metabolism. The device can even be employed as a neural implant to manage neurodegenerative diseases, where prolonged controlled drug delivery would serve to significantly enhance patient quality of life.

\section{REFERENCES}

1. du Toit LC, Pillay V, Choonara Y, Pillay S, Harilall S. Patenting of nanoparticles in drug delivery: No small issue. Recent Patents on Drug 
Delivery and Formulations 1 2007; Number 2: 131-142.

2. Ganta S, Devalapally H, Shahiwala A, Amiji M. A review of stimuli-responsive nanocarriers for drug and gene delivery. Journal of Controlled Release 2008; 126: 187-204.

3. Subbiah R, Veerapandian M, Yun KS, Nanoparticles: Functionalization and multifunctional applications in biomedical sciences. Current Medicinal Chemistry 2010; 17: 4559-4577.

4. Hughes GA. Nanostructure-mediated drug delivery. Nanomedicine: Nanotechnology, Biology and Medicine 2005; 1: 22-30.

5. Roney C, Kulkarni P, Arora V, Antich P, Bonte F, Wu A, Mallikarjuana NN, Manohar S, Liang H, Kulkarni AR, Sung H, Sairam M, Aminabhavi TM. Targeted nanoparticles for drug delivery hrough the blood-brain barrier for Alzheimer's disease. Journal of Controlled Release 2005; 108: 193-214.

6. Pison U, Welte T, Giersig M, Groneberg DA. Nanomedicine for respiratory disease. European Journal of Pharmacology 2006; 533: 341-350.

7. Popovic N, Brundin P. Therapeutic potential of controlled drug delivery systems in neurodegenerative diseases. International Journal of Pharmaceutics 2006; 314: 120-126.

8. Reis CP, Neufeld RJ, Ribeiro AJ, Veiga F. Nanoencapsulation II. Biomedical applications and current status of peptide and protein nanoparticulate delivery systems. Nanomedicine: Nanotechnology, Biology and Medicine 2006; 2: 53-65

9. Douglas KL, Piccirillo CA, Tabrizian M. Effects of alginate inclusion on the vector properties of chitosan-based nanoparticles. Journal of Controlled Release 2006; 115: 354-361.

10. Liu Z, Jiao Y, Wang Y, Zhou C, Zhang Z. Polysaccharides-based nanoparticles as drug delivery systems. Advanced Drug Delivery Reviews 2008; 60: 1650-1662.

11. Sriamornsak P, Nunthanid J, Luangtana-anan M, Weerapol Y, Puttipipatkhachorn S. Alginate-based pellets prepared by extrusion/spheronization: Effect of the amount and type of sodium alginate and calcium salts. European Journal of Pharmaceutics and Biopharmaceutics 2008; 69: 274-284.

12. Rajaonarivony $M$, Vauthier $C$, Couarraze G, Puisieux F, Couvreur P. Development of a new drug carrier made from alginate. Journal of Pharmaceutical Science 1993; 82: 912-917.

13. Li P, Dai Y, Zhang J, Wang A, Wei Q. ChitosanAlginate Nanoparticles as a Novel Drug Delivery System for Nifedipine International Journal of Biomedical Science 2008; 4: 221-228.

14. Li T, Shi X, Du Y, Tang Y. Quaternized chitosan/alginate nanoparticles for protein delivery. Journal of Biomedical Materials Research Part A 2007; 83A: 383-390.

15. Sarmento B, Ferreira DC, Jorgensen L, van de Weert M. Probing insulin's secondary structure after entrapment into alginate/chitosan nanoparticles. European Journal of Pharmaceutics and Biopharmaceutics 2007; 65: 10-17.

16. Zahoor A, Pandey R, Sharma S, Khuller GK. Pharmacokinetics and pharmacodynamic behaviour of antitubercular drugs encapsulated in alginate nanoparticles at two doses. International Journal of Antimicrobial Agents 2006; 27: 409416.

17. Zahoor A, Sharma S, Khuller GK. Inhalable alginate nanoparticles as antitubercular drug carriers against experimental tuberculosis. International Journal of Antimicrobial Agents 2005; 26: 298-303.

18. Guo J, Skinner GW, Harcum WW, Barnum PE. Pharmaceutical applications of naturally occurring water-soluble polymers Pharmaceutical Science and Technology Today 1998; 1: 254-261.

19. Lee J, Kim JS, Lee HG. $\gamma$-Oryzanol-loaded calcium pectinate microparticles reinforced with chitosan: Optimization and release characteristics. Colloids and Surfaces B: Biointerfaces 2009; 70: 213-217.

20. Song Y, Lee J, Lee HG. $\alpha$-Tocopherol-loaded Capectinate microcapsules: Optimization, in vitro release, and bioavailability. Colloids and Surfaces B: Biointerfaces 2009; 73: 394-398.

21. Biswal DR, Singh RP. Characterisation of carboxymethyl cellulose and polyacrylamide graft copolymer. Carbohydrate Polymers 2004; 57: 379 387.

22. Pushpamalar V, Langford SJ, Ahmad M, Lim YY. Optimisation of reaction conditions for preparing carboxymethyl cellulose from sago waste. Carbohydrate Polymers 2006; 64: 312-318.

23. Rokhade AP, Agnihotri SA, Patil SA, Mallikarjuna NN, Kulkarni PV, Aminabhavi TM. Semi-interpenetrating polymer network microspheres of gelatin and sodium carboxymethyl cellulose for controlled release of ketorolac tromethamine. Carbohydrate Polymers 2006; 65: 243-252.

24. Yuan N, Lin, Ho M, Wang, D, Lai J, Hsieh H. Effects of the cooling mode on the structure and strength of porous scaffolds made of chitosan, alginate, and carboxymethyl cellulose by the freeze-gelation method. Carbohydrate Polymers 2009; 78: 349-356.

25. Chang $\mathrm{K}$, Lee $\mathrm{Y}$. Ring Ring-opening polymerization of $\varepsilon$-caprolactone initiated by the antitumor agent doxifluridine. Acta Biomaterialia 2009; 5: 1075-1081.Bajpai SK, Sharma S. Investigation of swelling/degradation behaviour of alginate beads crosslinked with $\mathrm{Ca}^{+}$and $\mathrm{Ba}^{+}$ ions. Reactive and Functional Polymers 2004; 59: 129-140.

26. Huang M, Li Sand Vert M. Synthesis and degradation of PLA-PCL-PLA triblock copolymer prepared by successive polymerization of $\varepsilon$-caprolactone and DL-lactide. Polymer 2004; 45: 8675-8681.

27. Liu LS, Fishman ML, Hicks KB, Kende M. Interaction of various pectin formulations with 
porcine colonic tissues Biomaterials 2005; 26 : 5907-5916.

28. Luong-Van E, Grondahl L, Ngiap Chua K, Leong $\mathrm{KW}$, Nurcombe V, Cool SM. Controlled release of heparin from poly( $\varepsilon$-caprolactone) electrospun fibers. Biomaterials 2006; 27: 2042-2050.

29. Crowley MM, Zhang F, Koleng JJ, McGinity JW. Stability of polyethylene oxide in matrix tablets prepared by hot-melt extrusion. Biomaterials 2002; 23: 4241-4248.

30. Maggi L, Segale L, Torre ML, Ochoa Machiste E, Conte U. Dissolution behaviour of hydrophilic matrix tablets containing two different polyethylene oxides (PEOs) for the controlled release of a water-soluble drug. Dimensionality study. Biomaterials 2002; 23: 1113-1119.

31. Rezwan K, Chen QZ, Blaker JJ, Boccaccini AR. Biodegradable and bioactive porous polymer/inorganic composite scaffolds for bone tissue engineering. Biomaterials 2006; 27: 34133431 .

32. McKenzie JL, Waid MC, Shi R, Webster TJ. Decreased functions of astrocytes on carbon nanofiber materials. Biomaterials 2003; 25: 13091317.

33. Crowley MM, Fredersdorf A, Schroeder B, Kucera S, Prodduturi S, Repka MA, McGinity JW. The influence of guaifenesin and ketoprofen on the properties of hot-melt extruded polyethylene oxide films. European Journal of Pharmaceutical Sciences 2004; 22: 409-418.

34. Neto CGT Dantas TNC, Fonseca JLC, Pereira MR. Permeability studies in chitosan membranes. Effects of crosslinking and poly(ethylene oxide) addition. Carbohydrate Research 2005; 340: 26302636.
35. Don T, Huang M, Chiu A, Kuo K, Chiu W, Chiu L. Preparation of thermo-responsive acrylic hydrogels useful for the application in transdermal drug delivery systems. Materials Chemistry and Physics 2008; 107: 266-273.

36. Liu P, Zhai M, Li J, Peng J, Wu J. Radiation preparation and swelling behaviour of sodium carboxymethyl cellulose hydrogels. Radiation Physics and Chemistry 2002; 63: 525-528.

37. Parolis LAS, van der Merwe R, Groenmeyer GV, Harris PJ. The influence of metal cations on the behaviour of carboxymethyl cellulose as a talc depressant. Colloids and Surfaces A: Physicochemical and Engineering Aspects 2008; 317: 109-115.

38. Bajpai S.K and Sharma S, Investigation of swelling/degradation behaviour of alginate beads crosslinked with $\mathrm{Ca}^{2+}$ and $\mathrm{Ba}^{2+}$ ions, (Reactive and Functional Polymers), 59, Issue 2, (2004), pp. 129-140.

39. Zactiti EM, Kieckbusch TG. Potassium sorbate permeability in biodegradable alginate films: Effects of the antimicrobial agent concentration and crosslinking degree. Journal of Food Engineering 2006; 77: 46-467.

40. Oh SY, Jeong SY, Park TG, Lee JH. Enhanced transdermal delivery of AZT (Zidovudine) using iontophoresis and penetration enhancer. Journal of Controlled Release 1998; 51: 161-168.

41. Muthu MS, Rawat MK, Mishra R, Singh S. PLGA nanoparticle formulations of risperidone: Preparation and neuropharmacological evaluation. Nanomedicine: Nanotechnology, Biology and Medicine 2009; 5: 323-333. 